\title{
Research on a modified carrier capture based on frequency analysis
}

\section{Zhu Yuming, Wu Gangzhi, Fu Gang}

China Satellite Maritime Tracking and Control Department, Jiangyin, 214431, China

Keywords: Spectrum analysis; carrier acquisition; frequency estimation

\begin{abstract}
Eliminate the impact of information and data for the hopping carrier spectrum analysis based on parallel acquisition algorithm using trigonometric function transform method using three-point quadratic interpolation to refine the frequency estimate. By this method improved to improve the accuracy of the Doppler capture, to further improve the accuracy of carrier acquisition. FPGA design and implementation of the program provides a theoretical basis.
\end{abstract}

\section{Introduction}

In the study of signal acquisition algorithm should also consider some of the monitoring and control of its own characteristics. In the field of measurement and control, spacecraft trajectories are known, in most cases you can use the spacecraft trajectories in terms of the spacecraft motion brought by the Doppler effect, but will also appear Spacecraft on track the situation, therefore should be considered in the capture of the optimal conditions for capture unknown Doppler algorithm and under known conditions Doppler algorithm for optimal capture.

\section{Overall concept and design diagram}

In the measurement and control system, sometimes in a very short period of time the system needs to inject large amounts of remote data, monitoring and control systems to capture the spread should be able to meet the rapid capture of information at high speed conditions, and the high rate of problems brought on information as we talked about above. If only by coherent accumulation method to improve the system processing gain, capture system in the case of multiple data transitions is difficult to complete effective estimate carrier frequency and phase of the pseudo-code, so the need for carrier-based carrier spectrum analysis of parallel algorithms to improve capture . Trigonometric function transform coherent and non-coherent accumulation accumulate a combination of methods can reduce the impact of data transitions, and effectively improves the processing gain of the system. Achieve diagram shown in Figure 1.



Figure 1. FFT carrier frequency estimation algorithm Block Diagram

\section{Trigonometric transform information data offset the impact hopping}

Signal received by the receiver may be expressed as: 


$$
\operatorname{Re}\left(i T_{s}\right)=\sqrt{2 P} D\left(i T_{s}-\tau\right) P N\left(i T_{s}-\tau\right) \cos \left[2 \pi\left(f_{0}+f_{d}\right) i T_{s}+\phi_{0}\right]+n(i)
$$

Suppose the phase symbol of the input signal delay and Doppler shift in the integration accumulated time remains unchanged, the low-pass, after the output of the integrator accumulates as follows:

$$
\begin{aligned}
& I(k)=\sqrt{2 P} A D(k) R(\rho(k)) \sin c\left[\pi T \Delta f_{d}(k)\right] \cos \left[\Delta f_{d}(k) k T+\varphi_{0}\right]+H_{1}(k) \\
& Q(k)=-\sqrt{2 P} A D(k) R(\rho(k)) \sin c\left[\pi T \Delta f_{d}(k)\right] \sin \left[\Delta f_{d}(k) k T+\varphi_{0}\right]+H_{Q}(k)
\end{aligned}
$$

After subsequent data conversion, assuming $A^{\prime}=\sqrt{2 P} A R(\rho(k)) \sin c\left[\pi T \Delta f_{d}(k)\right]$, then the new I, Q two signals becomes:

$$
\begin{aligned}
& I^{\prime}(k)=A^{\prime 2} D^{2}(k) \cos \left[2 \Delta f_{d}(k) k T+2 \varphi_{0}\right]+H_{\rho}^{\prime}(k) \\
& Q^{\prime}(k)=A^{\prime 2} D^{2}(k) \sin \left[2 \Delta f_{d}(k) k T+2 \varphi_{0}\right]+H_{Q}^{\prime}(k)
\end{aligned}
$$

Wherein, $A_{\rho}^{\prime}(k)$ and $A_{g}^{\prime}(k)$ are mixed after the noise, the specific values are discussed below. Through these new I', Q' two-way analytic signal spectrum analysis, the estimated position of $\Delta f_{d}$.

Set IF carrier is $70 \mathrm{MHz}$, the code length is 1023 , the pseudo-code rate $10.23 \mathrm{Mchip} / \mathrm{s}$, sampling rate $56 \mathrm{MHz}$, after down-conversion low-pass filter 2 times extraction, a single point of accumulating 80 sampling points was made after 2048 points FFT, frequency resolution $342 \mathrm{~Hz}$, dynamic frequency estimation range $180 \mathrm{kHz}$. Suppose the Doppler shift of the received signal is set to $75.5 \mathrm{kHz}$, a pseudo-code modulation cycle one bit of data. The simulation shown in Figure 2.

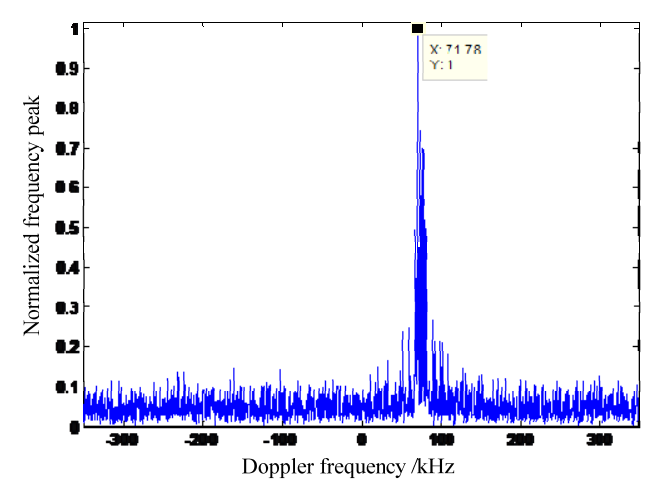

(a) Traditional carrier frequency estimate

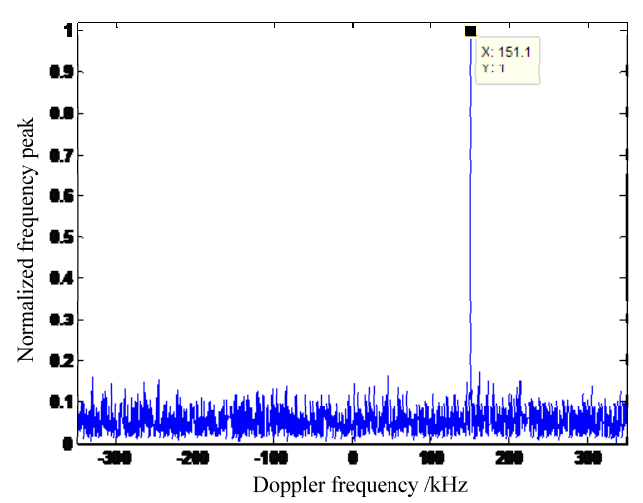

(b) Improved carrier frequency estimate

Figure 2. In the case of data transitions, the two frequency estimation algorithm schematic

Through the above chart it can be concluded, in the case of high bit rate, if you use the classic carrier frequency estimation method, it is difficult to estimate the exact frequency, and there is a higher side lobes. But if you use trigonometric function transform method to estimate the carrier frequency, then you can ignore the problem of data transitions, thus using the integral features to improve the frequency estimation accuracy.

\section{Three quadratic interpolation auxiliary carrier frequency estimation}

In capture more accurate frequency estimate, with a late fast catching PLL can be designed to track the more narrow, the target speed value is more accurate estimate. The existing research can know, due to various conditions, through improved frequency resolution FFT algorithm is more difficult to refine, can be found through simulation, can further improve the accuracy of frequency estimation using mathematical principle of three quadratic interpolation.

Three quadratic interpolation method principles:

Three quadratic interpolation method and frequency peaks around two treatment methods based on peak times, the first to establish a quadratic polynomial interpolation.

$$
y=a_{0} x^{2}+a_{1} x+a_{2}
$$


The three data points corresponding values into the formula,

$$
\left\{\begin{array}{l}
y_{0}=a_{0} x_{0}^{2}+a_{1} x_{0}+a_{2} \\
y_{1}=a_{0} x_{1}^{2}+a_{1} x_{1}+a_{2} \\
y_{2}=a_{0} x_{2}^{2}+a_{1} x_{2}+a_{2}
\end{array}\right.
$$

Can be obtained by solving the equations:

$$
\left\{\begin{array}{l}
a_{0}=\left(A_{1} C_{0}-A_{0} C_{1}\right) /\left(A_{1} B_{0}-A_{0} B_{1}\right) \\
a_{1}=\left(B_{1} C_{0}-B_{0} C_{1}\right) /\left(A_{0} B_{1}-A_{1} B_{0}\right)
\end{array}\right.
$$

Formula, $A_{0}=x_{0}-x_{1}, B_{0}=x_{0}^{2}-x_{1}^{2}, C_{0}=y_{0}-y_{1}, A_{1}=x_{0}-x_{2}, B_{1}=x_{0}^{2}-x_{2}^{2}, C_{1}=y_{0}-y_{2}$

Due to the above formula is a parabola, while according to the characteristic three data points available this parabola has a maximum value. Then when $\frac{\partial y}{\partial x}=0, \mathrm{x}$ corresponds to the maximum value. Is the exact location of spectral peaks, set $P_{Q}$, then there is the following expression:

$$
P_{Q}=-\frac{a_{1}}{2 a_{0}}
$$

Through the FFT frequency point quadratic fit, thereby achieve a better estimate of the Doppler frequency.

The following two experiments through simulation to illustrate its performance.

The simulation (a)

Estimate the dynamic frequency error. Simulation conditions: pre-Doppler frequency is $133 \mathrm{kHz}$, dynamic Doppler range is $130 \mathrm{kHz} \sim 135 \mathrm{kHz}, \mathrm{SNR}$ is $50 \mathrm{~dB}-\mathrm{Hz}$, the fundamental frequency FFT output is $2187.5 \mathrm{~Hz}$.

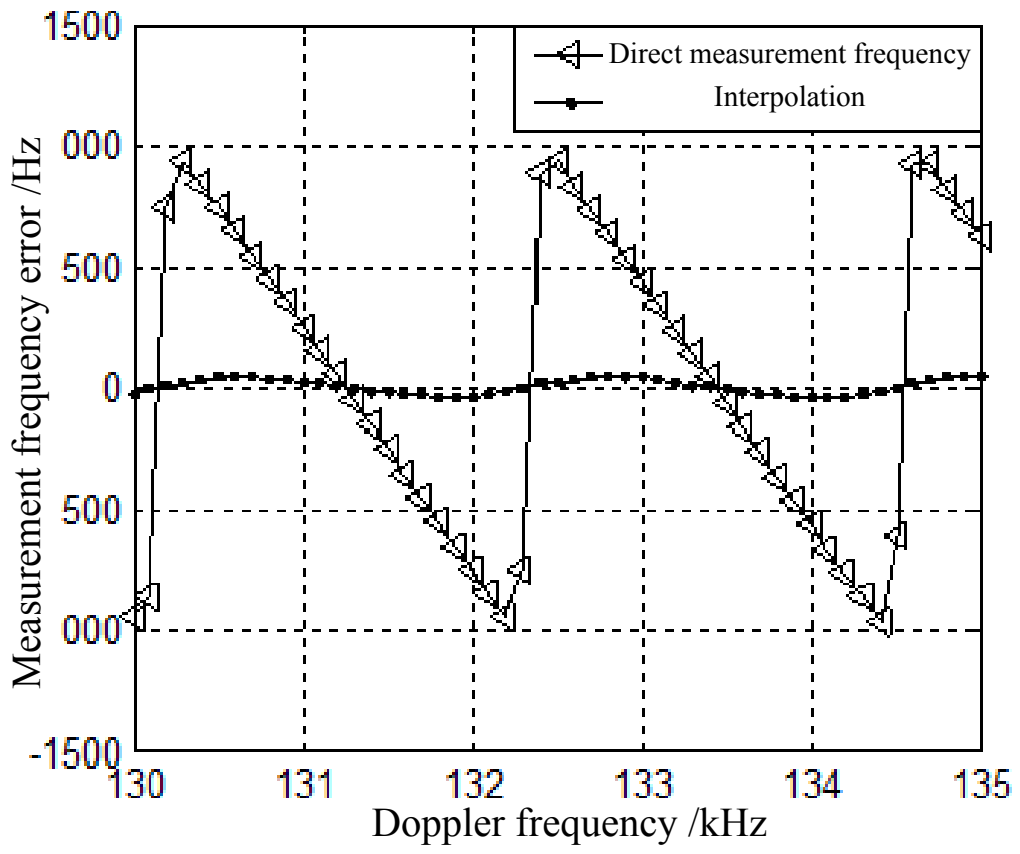

Figure 3. The frequency error or without interpolation frequency assisted

The simulation (b)

Using a band-pass Gaussian white noise affecting the statistical noise to capture accuracy. By the carrier to noise ratio under different carrier error capture algorithm to analyze noise immunity, and has a frequency estimation error contrast binomial interpolation auxiliary and ancillary situation goes items. Simulation conditions: Pseudo-code rate 10.23Mchip / s, the Doppler frequency of 150 $\mathrm{kHz}$. Under each carrier to noise ratio conditions, simulation of 1000, the statistical error. 


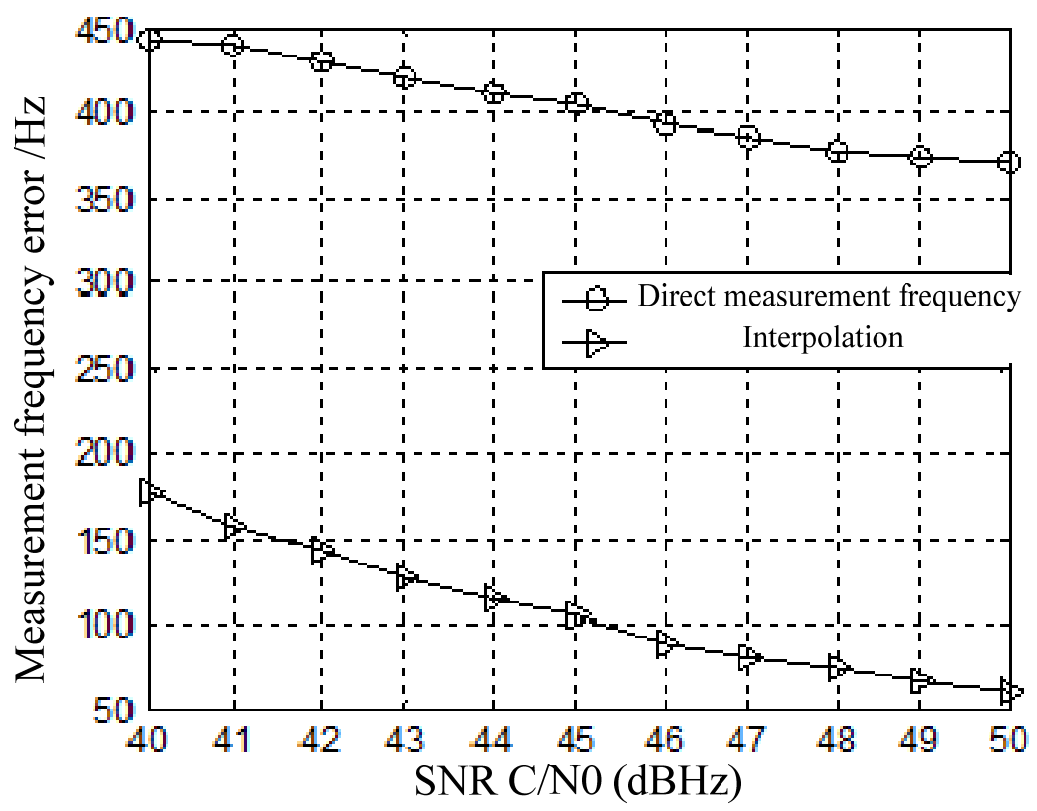

Figure 4. Different carrier to noise ratio and without interpolation frequency frequency error-assisted

After a lot of statistics simulation test and found that at high SNR conditions, the three-point quadratic interpolation frequency estimation error is $1 / 16$ of the original FFT estimation error at low SNR, quadratic interpolation error still reach $1 / 2$ of the original estimation error. It can be used for engineering applications.

\section{Conclusion}

This improvement based carrier spectrum analysis captured parallel algorithm, using data conversion and control data offset the impact of the captured data to solve the contradiction between the carrier frequency hopping between estimates. Quadratic interpolation using three auxiliary carrier frequency estimation precision Doppler capture, to further improve the accuracy of carrier capture.

\section{Reference}

[1] J.K Holmes, C Chen. Acquisition time performance of PN spread-spectrum systems[J].IEEE Transactions on communications,2011:778-784.

[2] R.A.Iltis. Joint estimation of PN code delay and multipath using the Extended Kalman Filter[J],IEEE Transactions on communications.2010:1677-1685.

[3] R.A.Iltis. An EKF-based joint estimator for interference multi-path, and code delay in a DS spread-spectrum receiver[J],IEEE Transactions on communications.2013:1288-1299.

[4] M.G.El-tarhhuni and U.H.Sheikh. Application of adaptive filtering to direct-sequence spread-sprectrum code acquisition[J], Wireless Personal Communication.2008:185-204 